\title{
Evaluation of $A L M 5$ and MMP13 Expression in the Cartilage Tissue of Rats with Osteoarthritis Rats and Effects of Exercise Training, Ozone and Mesenchymal Stem Cell Therapies on Expression of these Genes
}

\author{
Samira Oladazimi \\ Department of Exercise Physiology, \\ Faculty of Humanities, Sari Branch, \\ Islamic Azad \\ University, Sari, Iran \\ Parvin Farzanegi (PhD) \\ Department of Exercise Physiology, \\ Faculty of Humanities, Sari Branch, \\ Islamic Azad \\ University, Sari, Iran \\ Mohammad Ali Azarbayejani (PhD) \\ Department of Exercise Physiology, \\ Islamic Azad University, Central \\ Tehran Branch \\ Corresponding author: Parvin \\ Farzanegi \\ Tel: +989112230233 \\ Email: parvin.farzanegi@gmail.com \\ Address: Department of Exercise \\ Physiology, Faculty of Humanities, \\ Sari Branch, Islamic Azad; University, \\ Sari, Iran \\ Received: 07 Jan 2018 \\ Revised: 20 Jan 2019 \\ Accepted: 22 Jan 2019

\section{(c) (7) (8)} \\ This work is licensed under a Creative \\ Commons Attribution 4.0 License.
}

\begin{abstract}
IBSTRACT
Background and objectives: Matrix metalloproteinase-13 (MNP-13) and activin receptor-like kinase 5 (ALK5) are considered as important factors contributing to knee osteoarthritis $(0 \mathrm{~A})$ pathogenesis. Here, we compared therapeutic effects of mesenchymal stem cells (IISCS), ozone and exercise training alone and combined on expression of MMP-13 and ALK5 in rats with knee $0 \mathrm{~A}$.

Methods: Knee 0A was induced by a surgical method. Rats with $0 \mathrm{~A}$ were then randomly divided into several groups, including model, MSCs, ozone, exercise, MSCs + ozone, MSC's + exercise, ozone + exercise and MSC's + ozone + exercise groups. Expression of MMP-13 and ALK5 genes was evaluated using RT-PCR. Data were analyzed using SPSS software at significance of 0.05 .

Results: Expression of MMP-13 and ALK5 differed significantly between the study groups $(\mathrm{P}<0.0001)$. Knee $0 \mathrm{~A}$ was significantly associated with overexpression of MMP-13 and ALK5 in the cartilage tissue of rats with knee 0A. Combined therapy with MSCs, ozone and exercise significantly decreased the expression of MMP-13 and ALK5 in the cartilage of rats with $0 \mathrm{~A}(\mathrm{P}<0.001)$. Although MSCs, ozone and exercise training were effective to mitigate expression of MMP-13 and ALK5 genes, ozone therapy was more effective compared to the other two therapies.

Conclusion: Although ozone, MSC's and exercise training alone could decrease the expression of MMP-13 and ALK5 genes, combined therapy with MSC, ozone and exercise is more effective.
\end{abstract}

Keywords: 0ste0arthritis, 03, MSCL, exercise, MMP-13, ALK5.

This paper should be cited as: Oladazimi S, Farzanegi P, Azarbayejani MA [Evaluation of ALK5 and MMP13 Expression in the Cartilage Tissue of Rats with Osteoarthritis Rats and Effects of Exercise Training, Ozone and Mesenchymal Stem Cell Therapies on Expression of these Genes ]. mljgoums. 2020; 14(1): 50-56 


\section{INTRODUCTION}

Osteoarthritis (OA) is the most common form of articular disease that causes chronic joint pain, swelling and stiffness, particularly in knees and feet. Approximately 30 million people in the United States suffer from OA (1). The incidence of knee OA is reported to be significantly increased since the 20th century (1). Several factors such as obesity, genetic factors, diabetes, low physical activity, and walking disability are contributed to OA development and progression $(2,3)$.

Despite the high incidence of knee OA throughout the world, there is no approved medication or treatment strategy for the disease control and current treatments are focused on amelioration of symptoms and chronic pain. Therefore, numerous studies have investigated the cellular and molecular mechanisms of $\mathrm{OA}$ pathogenesis and its potential therapeutic strategies. Recent studies have indicated that multiple signaling pathways are probably involved in the disease development and progression. Matrix metalloproteinases (MMPs) are a class of zincdependent endopeptidases which are involved in the degradation of extracellular matrix proteins, such as collagen (4). Although MMPs are responsible for various physiological and developmental processes, abnormal expression of these enzymes can be associated with pathological events and tissue damage (5). Several factors such as interleukin-1 (IL-1), IL-6, tumor necrosis factor- $\alpha$, transforming growth factor (TGF- $\beta$ ), epidermal growth factor, and basic fibroblast growth factor enhance the expression of MMPs, while corticosteroids and IL-4 decrease MMPs expression (6). More than 20 MMPs have been identified in mammalian cells which are categorized based on their functions.

MMP-13 is a member of the MMPs family that plays a critical role in the degradation of fibrillar collagen and fibronectin. Some studies demonstrated that overexpression of MMP-13 is a significant factor for OA development and progression (7). For this reason, recent studies indicated that pharmacologic inhibition of MMP13 may be an effective strategy to ameliorate articular cartilage loss in patients with knee OA (7). In addition to MMP-13, activin receptor-like kinase 5 (ALK5), also known as TGF- $\beta$ receptor type $\mathrm{I}$, might be involved in OA development (8) and is required for TGF $\beta$-initiated chondrogenic differentiation (9).

Given the critical role of MMP-13 and ALK5 in OA development and progression, they can be considered as potential targets for OA therapy. Recent studies have demonstrated that ozone therapy can be used safely for treatment of OA (10). For instance, Feng et al. showed that ozone therapy improved knee pain in patients with OA (11). In addition to ozone therapy, mesenchymal stem cells (MSCs) have been recommended for cartilage repair and knee OA treatment (12). Due to their immunomodulatory properties, MSCs have been studied for treatment of various diseases such as cancer, arthritis, diabetes and autoimmune diseases. Physical activity or exercise training is another therapeutic strategy which has been suggested for the treatment of knee OA. Several studies have demonstrated the positive effects of exercise training on OA features, such as pain, walking disability, and stair climbing in patients with knee OA (13). Although these studies reported the effectiveness of exercise training, ozone and MSC therapies for OA treatment, the cellular and molecular mechanisms of such effects are not clear. Since MMP-13 and ALK5 play central roles in OA development, we assumed that MSC and ozone therapies along with exercise training may be effective for improvement of $M M P-13$ and ALK5 expression. Therefore, we designed this study to consider the effect of exercise training, MSCs and ozone therapies alone and combined on MMP-13 and ALK5 expression in rats with knee $\mathrm{OA}$.

\section{MATERIALS AND METHODS}

Sixty-three male Wistar rats (aged 40 to 45 weeks) weighing 250-300g were obtained from the laboratory animal research center at Islamic Azad University of Sari, Iran. The animals were housed 3 per cage $(42 \times 26.5 \times$ $15 \mathrm{~cm}$ ) in a climate-controlled room (ambient temperature of $22 \pm 2{ }^{\circ} \mathrm{C}$, humidity of $50 \pm 5$, and under a 12:12 light/dark cycle).

Furthermore, all rats were fed with a standard diet and water. The study protocol on experimental rats was approved by the animal care and use committee at Islamic Azad University, Sari branch, Iran (ethical number: NO.19.33.2018). Knee OA in rats was induced by a surgical method (14). 
Briefly, the rats were anesthetized with ketamine $(30-50 \mathrm{mg} / \mathrm{kg}$ ) and xylasine (3-5 $\mathrm{mg} / \mathrm{kg}$ ), and a $1 \mathrm{~cm}$ longitudinal incision was made to expose knee joint in a sterilized environment. Knee joint was immediately opened through lateral dislocation of the patella and patellar ligament. A longitudinally cut was made in knee joint capsule and then an incomplete incision was made through the medial meniscotibial ligament without articular cartilage and other ligaments injury. Eventually, the knee joint capsule was closed with a 6-0 absorbable suture and the skin was closed with 6-0 silk suture. One drop of $0.25 \%$ bupivacaine was used to minimize postoperative pain in the surgical site. Rats were fed with standard food and water for three weeks. Rats with knee OA were then randomly divided into eight groups including: model (knee OA rats without any further treatments), MSCs, ozone, exercise, MSCs + ozone, MSCs + exercise, ozone + exercise, and MSCs + ozone + exercise groups. Control rats were healthy and did not receive any of the above therapies. Rats in the combined therapy groups initially received MSC therapy, and then ozone therapy and finally exercise training.

Mesenchymal stem cells were purchased from the Histogenotech Company (Tehran, Iran). According to the company's instructions, bone marrow-derived MSCs were extracted from healthy male Wistar rats (weighing 25-300 g). The purchased MSCs were cultured in DMEM medium with $20 \%$ fetal bovine serum and incubated overnight to select for adherent cells. The culture medium in culture flasks was changed every three days and MSCs were passaged 3-4 times. After MSCs reached

$>90 \%$ confluency, they were selected for injection purposes. Rats in the MSCs groups received intraarticular injection of $1 \times 10^{6}$ cells $/ \mathrm{kg}$.

Ozone was generated by OZOMED 01 equipment from medical-grade oxygen and through a silent electric discharge. Its concentration was measured using a UV spectrophotometer at $254 \mathrm{~nm}$. Ozone was injected into the knee through the tibiofemoral joint line at concentration of $20 \mu \mathrm{g} / \mathrm{ml}$, once weekly for three weeks starting at 21 days after the modeling.

The rats were familiarized with treadmill running for one week (three times per week, with $\mathrm{VO}_{2} \mathrm{max}$ of $60-70 \%$, speed of $16 \mathrm{~m} / \mathrm{min}$, and zero inclination for $10 \mathrm{~min} /$ day) (15). The aerobic exercise training was then initiated with a 30-minute run on the treadmill without slope at speed of $16 \mathrm{~m} / \mathrm{min}$ in the first week. The duration was gradually increased to 50 minutes by the eighth week. Warm-up and cool-down were done at $5 \mathrm{~m} / \mathrm{min}$ in the beginning and at the end of each exercise session.

Forty-eight hours after the final training session, the rats in each group were anesthetized with ketamine $(30-50 \mathrm{mg} / \mathrm{kg}$ ) and xylasine $(3-5 \mathrm{mg} / \mathrm{kg})$. Cartilage tissue of the knees was isolated and used for gene expression analysis.

Cartilage tissues were homogenized in phosphate buffer saline (0.01 M; pH 7.0) at 4 ${ }^{\circ} \mathrm{C}$ with a homogenizer (Hielscher, UP100H). Total RNA was extracted using the RNX-Plus kit (SinaClon; RN7713C). The quantity and quality of the extracted RNA were evaluated using the Nanodrop ND-1000 spectrophotometer (Thermo Sci., Newington, $\mathrm{NH})$. Then, RNA samples were transcribed to cDNA using Revert Aid Reverse Transcriptase (Thermo scientific, Germany) at $42^{\circ} \mathrm{C}$ for $1 \mathrm{~h}$, using random hexamer primers (Thermo scientific, Germany). A Rotor Gene 6000 (Corbett Research, Australia) thermocycler and Real Q-PCR 29 Master Mix Kit (Amplicon, Denmark) were applied for amplifications in 40 cycles. Each reaction solution included $5 \mu \mathrm{l}$ of master mix and 100 nmol of primers. The primers were designed based on published data and the NCBI database (http://www.ncbi.nlm.nih.gov/build 131). Primer sequences are summarized in table 1. Glyceraldehyde 3-phosphate dehydrogenase (GAPDH) was applied as a reference gene. The mRNA level of $M M P 13$ and $A L K 5$ was normalized relative to the amount of GAPDH mRNA.

Expression of $M M P 13$ and $A L K 5$ mRNAs are presented as mean \pm standard deviation (SD). Comparison of the mean expression level of these genes between the study groups was performed using one-way ANOVA and post hoc-Tukey test. SPSS software (version 19, IBM) was used for data analysis. A p-value of less than 0.05 was considered as statistically significant.

\section{RESULTS}

Mean expression level of $M M P 13$ in all groups is presented in figure 1 . A significant difference was observed in the expression of 
MMP13 between the groups $(\mathrm{P}<0.001)$. Therapy with MSCs + ozone + exercise and MSCs + ozone significantly decreased the expression of MMP13 in the cartilage of OA rats compared to the other groups. In addition, MMP13 expression was significantly higher in the model group compared to other groups $(\mathrm{P}<0.001)$. Although MSCs, exercise and ozone therapies alone significantly decreased the expression of MMP13 compared to the model group $(\mathrm{P}<0.001)$, their combination was more effective. Ozone therapy was significantly more effective to mitigate $M M P 13$ expression compared to MSC therapy and exercise training $(\mathrm{P}<0.01)$.

Mean level of $A L K 5$ expression in all study groups is shown in figure 2. The model and exercise groups had the highest level of ALK5 expression, while combined therapy with ozone and MSCs significantly decreased the expression of $A L K 5$ compared to other groups $(\mathrm{P}<0.001)$.

Although MSC and ozone therapies alone decreased the expression of ALK5, their combination was more effective. Ozone therapy was significantly more effective than MSC therapy $(\mathrm{P}<0.01)$.

Table 1- Primers sequence for studied genes

\begin{tabular}{ccc}
\hline Gene name & & Sequences \\
\hline$M M P 13$ & Forward & 5/-TGGAGTGCCTGATGTGGGTGA-3/ \\
& Reverse & 5/- TGGAGTGCCTGATGTGGGTGA-3/ \\
ALK5 & Forward & 5/-ATGTTGGAGATAGTGATGTTG-3/ \\
& Reverse & 5/-TGGTAGGTTTGAGATAAGATG-3/ \\
Sirt1 & Forward & 5'-GAGTTGTGTGTAGGTTAGGTGG-3' \\
& Reverse & 5'-AAATATGAAGAGGTGTTGGTGG-3' \\
GAPDH & Forward & 5'-AAGTTCAACGGCACAGTCAAGG-3' \\
& Reverse & 5'-CATACTCAGCACCAGCATCACC-3 \\
\hline
\end{tabular}

Figure 1- Comparison of the mean mRNA levels of MMP13 between the study groups. Gene expression was detected by RT-PCR. There was no significant difference in the mRNA levels of MMP13 between groups with similar symbols (a-f). The mean mRNA level of $M M P 13$ was in this order: $a>b>c>d>e>f$. MSC: mesenchymal stem cell; Exp: exercise

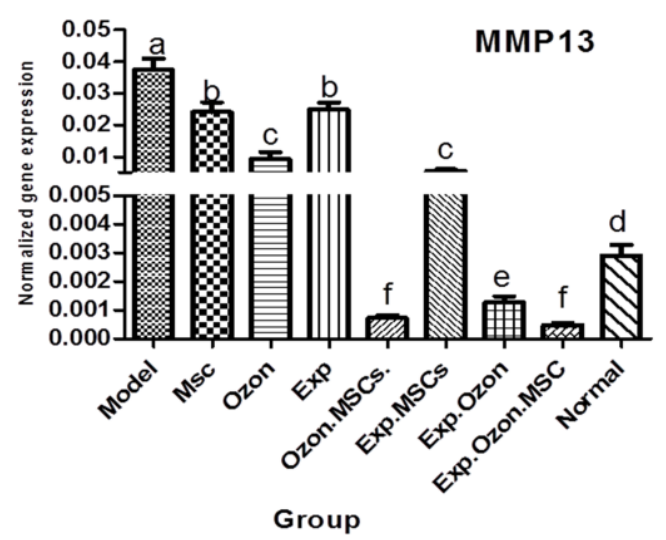

Figure 2- Comparison of the mean mRNA levels of $A L K 5$ between the study groups. Gene expression was detected by RT-PCR. There was no significant difference in the mRNA level of $A L K 5$ between the groups with similar symbols (a-f). The mean mRNA level of $A L K 5$ was in this order: $a>b>c>d>e>f$. MSC: mesenchymal stem cell; Exp: exercise

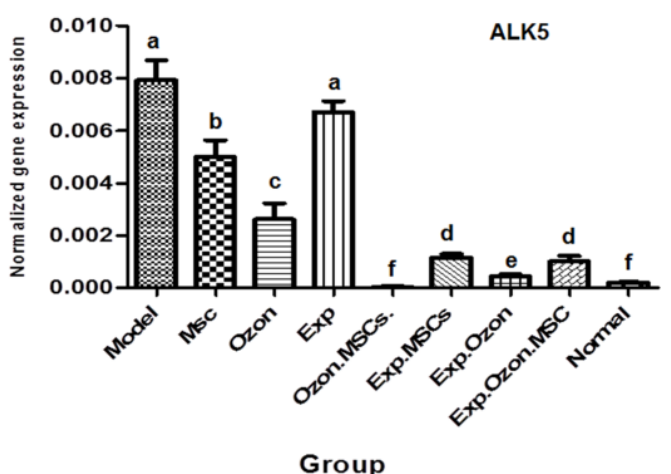




\section{DISCUSSION}

In this study, we examined the effects of exercise training, ozone and MSC therapies alone or combined on expression of MMP13 and ALK5 genes in the cartilage tissue of rats with knee OA. Our data show that knee OA was associated with a significant increase in $M M P 13$ and $A L K 5$ expression in cartilage cells of rats with OA, which is in agreement with previous studies $(7,16)$. In this line, Kamekura et al. demonstrated that overexpression of $M M P-13$ is significantly correlated to the presence of pathological chondrocytes and OA development in rats (17). Similarly, Sato et al. found increased expression of $M M P-13$ during the early stages of OA in humans (18). We found overexpression of $A L K 5$ in the cartilage of rats with knee OA. However, this is inconsistent with previous studies reporting that ALK5 deletion or downregulation is associated with increased risk of OA. A recent study has revealed that cartilage-specific deletion of ALK5 is associated with increased risk of OA-like phenotype in mice (19). Blaney et al. indicated that knockdown of ALK5 in chondrocytes induces MMP13 mRNA expression. They also reported a close relationship between ALK5 and ALK1 balance and $M M P-13$ expression (8). These data suggest that any change in normal expression pattern of ALK5 can be associated with abnormal expression of MMP13 and subsequently pathological events. Therefore, normal expression of $A L K 5$ and $M M P-13$ is necessary for the maintenance of articular cartilage homeostasis, while the dysregulation of these genes can cause cartilage degeneration and increased risk of OA. Wang et al. showed that MMP13 inhibition decelerates articular cartilage loss in a murine model of injuryinduced knee OA (7). A previous study showed that $M M P-13$ knockout in mice inhibits OA progression by protecting cartilage from proteoglycan loss and structural damage (20). To study the pathogenesis or therapeutic options for OA, we were the first to compare the effectiveness of exercise training, MSC and ozone therapies alone and combined on expression of ALK5 and MMP13 genes in the cartilage tissue of rats with knee OA. We found that these therapeutic methods, especially MSC and ozone therapies, significantly decreased the expression of ALK5 and MMP13 in the cartilage of rats with $\mathrm{OA}$; however, ozone therapy was more effective than MSC therapy alone. Furthermore, we found that combined therapy with MSCs and ozone was more effective to mitigate expression of ALK5 and MMP13 genes in the cartilage tissue of OA rats.

Some studies evaluated the effect of exercise training, ozone and MSC therapies on clinical and histopathological outcomes of OA. For example, de Jesus et al. investigated intraarticular effect of ozone therapy in treatment of knee OA. They found that ozone therapy significantly improved pain relief, function of knees, and quality of life in patients with knee OA (21). A more recent study has demonstrated that ozone therapy is associated with a significant improvement in hind-paw diameter, arthritis severity, and histopathological findings of inflammation in Wistar rats (22). Some studies have suggested that ozone therapy improves OA by improving the antioxidant defense system and decreasing oxidative damage and inflammatory responses caused by free radicals $(23,24)$. We assumed that decreased expression of ALK5 and MMP13 as another important mechanism of ozone therapy in OA treatment. However, further studies at protein levels are needed to confirm this theory. Some studies also reported that moderate exercise and MSC therapy increase $A L K 5$ and $M M P-13$ expression (25). In this line, Wang et al. reported that stretch exercise reduces the expression of $M M P-1$ and $M M P$ 13 (26). More recently, Yang et al. have demonstrated that treadmill exercise lowers MMP-13 expression in articular cartilage of rats with OA (27). In another study, MSC therapy significantly decreased expression of $M M P-13$ in rats with knee OA (28).

Our findings indicate that exercise training, MSC and ozone therapies are helpful for OA improvement. Moreover, decreased expression of $M M P-13$ and $A L K 5$ may be a possible mechanism of these therapies. Thus, these therapies may mitigate clinical and histopathological outcomes of arthritis by decreasing the expression of $M M P-13$ and ALK5 genes.

\section{CONCLUSION}

Our findings show that knee $\mathrm{OA}$ is strongly associated with increased expression of $A L K 5$ and $M M P-13$ in the cartilage tissue. Ozone therapy was more effective compared to the other therapies in reduction of 
expression of these gene and arthritis improvement. Although MSCs, ozone and exercise alone could decrease ALK5 and $M M P-13$ expression in the cartilage tissue of rats with OA, combined therapy with MSCs + ozone + exercise was significantly more effective.

\section{ACKNOWLEDGMENTS}

This research was supported by the REFERENCES

1. Wallace IJ, Worthington S, Felson DT, Jurmain RD, Wren KT, Maijanen H, et al. Knee osteoarthritis has doubled in prevalence since the mid-20th century. Proc Natl Acad Sci USA. 2017; 114: 9332- 6. doi: 10.3349/ymj.2015.56.1.124.

2. Lee KM, Chung CY, Sung KH, Lee SY, Won SH, Kim TG, et al. Risk Factors for Osteoarthritis and Contributing Factors to Current Arthritic Pain in South Korean Older Adults. Yonsei Med J. 2015; 56: 124-31. doi: 10.3349/ymj.2015.56.1.124.

3. Heidari B. Knee osteoarthritis prevalence, risk factors, pathogenesis and features: Part I. Caspian J Intern Med. 2011; 2(2): 205-12.

4. Sorsa T, Tjaderhane L, Salo T. Matrix metalloproteinases (MMPs) in oral diseases. Oral diseases. 2004; 10(6): 311-8.

5. Jablonska-Trypuc A, Matejczyk M, Rosochacki S. Matrix metalloproteinases (MMPs), the main extracellular matrix (ECM) enzymes in collagen degradation, as a target for anticancer drugs. J Enzyme Inhib Med Chem. 2016; 31(sup1): 177-183.

6. Lu L, Zhang Q, Pu LJ, Peng WH, Yan XX, Wang LJ, et al. Dysregulation of matrix metalloproteinases and their tissue inhibitors is related to abnormality of left ventricular geometry and function in streptozotocininduced diabetic minipigs. Int J Exp Pathol. 2008; 89(2): 125-37. doi: 10.1111/j.1365-2613.2008.00579.x.

7. Wang M, Sampson ER, Jin H, Ke QH, Im HJ, Chen D. MMP13 is a critical target gene during the progression of osteoarthritis. Arthritis Res Ther. 2013; 15(1): R5. doi: 10.1186/ar4133.

8. Blaney Davidson EN, Remst DF, Vitters EL, van Beuningen $\mathrm{HM}$, Blom $\mathrm{AB}$, Goumans $\mathrm{MJ}$, et al. Increase in ALK1/ALK5 ratio as a cause for elevated MMP-13 expression in osteoarthritis in humans and mice. $\mathrm{J}$ Immunol. 2009; 182(12): 7937-45. doi: 10.4049/jimmunol.0803991.

9. de Kroon LM, Blaney Davidson EN, Narcisi R, van Beuningen HM, van Osch GL, van der Kraan PM. Activin receptor-like kinase ALK5 and ALK1 are both required for TGF $\beta$-initiated chondrogenic differentiation of mesenchymal stem cells. Osteoarthritis and Cartilage. 2015; 32: A79.

10. Manoto SL, Maepa MJ, Motaung SK. Medical ozone therapy as a potential treatment modality for regeneration of damaged articular cartilage in osteoarthritis. Saudi J Biol Sci. 2018; 25(4): 672-679. doi: 10.1016/j.sjbs.2016.02.002.
Exercise Physiology Center of Islamic Azad University, Sari Branch, Iran.

We would like to thank the staff of the Exercise Physiology Center of the University for their cooperation.

\section{CONFLICT OF INTEREST}

The authors declare no conflict of interest regarding publication of this manuscript.

11. Feng X, Beiping L. Therapeutic Efficacy of Ozone Injection into the Knee for the Osteoarthritis Patient along with Oral Celecoxib and Glucosamine. J Clin Diagn Res. 2017 Sep;11(9):UC01-UC03. doi: 10.7860/JCDR/2017/26065.10533.

12. Davatchi F, Sadeghi Abdollahi B, Mohyeddin M, Nikbin B. Mesenchymal stem cell therapy for knee osteoarthritis: 5 years follow-up of three patients. Int $\mathbf{J}$ Rheum Dis. 2016; 19: 219-25.

13. Nejati P, Farzinmehr A, Moradi-Lakeh M. The effect of exercise therapy on knee osteoarthritis: a randomized clinical trial. Med J Islam Repub Iran. 2015; 29: 186.

14. Zhao Y, Liu B, Liu C. Establishment of a Surgicallyinduced Model in Mice to Investigate the Protective Role of Progranulin in Osteoarthritis. J Vis Exp. 2014; (84): e50924. doi: 10.3791/50924.

15. Farzanegi P, Abbas Zadeh H, Kazemi M, Sabbaghian M, Shoeibi A, Nabipour R, et al. Effects of aerobic exercise on histopathology and toxicology of $\mathrm{ZnO}$ and nano $\mathrm{ZnO}$ in male rats. Toxicological \& Environmental Chemistry. 2018; 100(1): 103-114.

16. Li NG, Shi ZH, Tang YP, Wang ZJ, Song SL, Qian $\mathrm{LH}$, et al. New hope for the treatment of osteoarthritis through selective inhibition of MMP-13. Curr Med Chem. 2011; 18(7): 977-1001.

17. Kamekura S, Hoshi K, Shimoaka T, Chung U, Chikuda H, Yamada T, et al. Osteoarthritis development in novel experimental mouse models induced by knee joint instability. Osteoarthritis Cartilage. 2005; 13(7): 632-41.

18. Sato $\mathrm{T}$, Konomi $\mathrm{K}$, Yamasaki S, Aratani $\mathrm{S}$, Tsuchimochi $\mathrm{K}$, Yokouchi $\mathrm{M}$, et al. Comparative analysis of gene expression profiles in intact and damaged regions of human osteoarthritic cartilage. Arthritis Rheum. 2006; 54(3): 808-17.

19. Wang Q, Tan QY, Xu W, Qi HB, Chen D, Zhou S, et al. Cartilage-specific deletion of Alk5 gene results in a progressive osteoarthritis-like phenotype in mice. Osteoarthritis Cartilage. 2017; 25(11): 1868-79.

20. Little CB, Barai A, Burkhardt D, Smith SM, Fosang $\mathrm{AJ}$, Werb Z, et al. Matrix metalloproteinase 13-deficient mice are resistant to osteoarthritic cartilage erosion but not chondrocyte hypertrophy or osteophyte development. Arthritis Rheum. 2009; 60(12): 3723-33. doi: 10.1002/art.25002. 
21. Lopes de Jesus CC, dos Santos FC, de Jesus LMOB, Monteiro I, Sant'Ana MSSC, Trevisani VFM. Comparison between intra-articular ozone and placebo in the treatment of knee osteoarthritis: A randomized, double-blinded, placebo-controlled study. PloS one. 2017; 12: e0179185. doi: 10.1371/journal.pone.0179185.

22. Bozbaş Ct, Yilmaz M, Paşaoğlu E, Gürer G, İvgin R, Demirci B. Effect of Ozone in Freund's Complete Adjuvant-Induced Arthritis. Arch Rheumatol. 2018; 33(2): 137-42. doi: 10.5606/ArchRheumatol.2018.6371. 23. Motohashi H, Yamamoto M. Nrf2-Keapl defines a physiologically important stress response mechanism. Trends Mol Med. 2004; 10(11): 549-57.

24. Mawsouf M, Maha El-Sawalhi M, Martínez-Sánchez G, Hebatalla Darwish A. Effect of ozone therapy on redox status in experimentally induced arthritis. Revista Española de Ozonoterapia. 2011; 1(1): 32-43.

25. Nejad-Moghaddam A, Ajdary S, Tahmasbpour E RF, Panahi Y, Ghanei M. Immunomodulatory Properties of Mesenchymal Stem Cells Can Mitigate Oxidative Stress and Inflammation Process in Human Mustard Lung. Biochem Genet. 2016; 54: 769-83.
26. Wang P, Yang L, You X, Singh GK, Zhang L, Yan $\mathrm{Y}$, et al. Mechanical stretch regulates the expression of matrix metalloproteinase in rheumatoid arthritis fibroblast-like synoviocytes. Connect Tissue Res. 2009; 50: 98-109.

27. Yang Y, Wang Y, Kong Y, Zhang X, Bai L. The effects of different frequency treadmill exercise on lipoxin A4 and articular cartilage degeneration in an experimental model of monosodium iodoacetate-induced osteoarthritis in rats. PloS one. 2017; 12: e0179162.

28. Tang J, Cui W, Song F, Zhai C, Hu H, Zuo Q, et al. Effects of mesenchymal stem cells on interleukin-1 $\beta$ treated chondrocytes and cartilage in a rat osteoarthritic model. Mol Med Rep. 2015; 12(2): 1753-60. doi: 10.3892/mmr.2015.3645. 